\title{
Multi-Objective Optimization of Electric Multiple Unit Wheel Profile From Wheel Flange Wear Viewpoint
}

\author{
Dabin Cui ${ }^{\mathrm{a}, \mathrm{b}}$, Ruichen Wang ${ }^{\mathrm{c}}$, Paul Allen ${ }^{\mathrm{c}}$, Boyang An ${ }^{\mathrm{b}}$, Li Li ${ }^{\mathrm{a}}$, Zefeng Wen \\ a School of Mechanical Engineering, Southwest Jiaotong University, Chengdu 610031, China \\ $b$ Key Laboratory of High-speed Railway Engineering, Ministry of Education, Chengdu 610031, China; \\ c Institute of railway research, University of Huddersfield, Huddersfield HD1 3DH, UK; \\ d State Key Laboratory of Traction Power, Southwest Jiaotong University, Chengdu 610031, China
}

*Corresponding author, Tel: +86-15982365391; fax: +86-28-87600868, E-mail address: cdb1645@163.com

\begin{abstract}
The CRH1 train is one of the main commuter trains in China which is mostly operating on typical and high-speed lines. Previously, a high-speed car wheel profile was used on the CRH1 train, but it does not match well with the train suspension parameters and also causes the instability of the train on tangent track and large curved track. Therefore a new profile was designed as the replacement of the old one for the CRH1 train. However, the use of the new profile results in the serious wheel flange and rail gauge corner wear but it can provide better stability compared to the old profile. This paper first presents the evaluation of using the two profiles, and then a development of the wheel profile is objected in terms of both currently used profiles, which is not only to minimize the flange wear and also take the vehicle dynamic behavior into consideration. A multi-objective optimization method was, therefore, to propose for the minimization of the lateral force and the stability of wheelsets. The requirements of the wheel profile geometry are investigated through proposed optimization method. Finally, the profile satisfied the safety requirements of the vehicle have been provided by using the particle swarm optimization method. Furthermore, the evaluation of vehicle dynamic has been performed by using Multi-Body Simulation Software. The entire design process has been completed in a closed loop procedure programmed in MATLAB. The findings show that the developed profile after the optimization procedure is fairly acceptable for the requirements of the wheel-rail interface and dynamic behavior of CRH1 train.
\end{abstract}

Keywords: Multi-objective optimization; flange wear; wheel profile design; vehicle dynamic behavior 


\section{Introduction}

Trains running mile after mile put high demands on their wheels. Each year, the cost of maintenance and replacement of wheel/rail wear in current rails is as high cost as several billion RMB (¥) in China [1]. With a large number of electric multiple unit (EMU) train come into service, the quality of railway in service have been greatly improved in last decade. Meanwhile, wheel and rail wear has attracted much more attention by railway researchers and engineers. It can be assumed that the demand of high-speed wheels is more than 20 thousands per year [2]. If the wheel cannot suitably match the rail, the extra wear would be taken, and the normal operation could even be affected. The additional wear can be produced by the use of the unmatched wheel-rail profiles and exert negative influence on the operation of the train.

The wheel profile optimization is a long-standing research topic on railway. Since the Nineteenth Century, there has been a basic understanding of wheel shape and its self-steering principle [3]. Based on many years' experience of research and operation [4-8], the wheel profile has been changed from conical wheel profile to worn wheel profile, and therefore the wear of the wheel/rail was significantly reduced to an acceptable level. At present, most of wheel profiles used in EMU trains have followed this design concept. Nowadays, a plenty of design concepts and methods were presented for the optimization of the wheel profiles because of the rapid development of the numerical optimization techniques. Haque et.al [9] developed a new wheel profile design method to balance the stability on a straight line with the performance on curved line. Yamada and Sasaki et.al [10] designed a CS profile in terms of the measured rail profiles. Wu [11] proposed a concept of wheel profile design to systemically evaluate the compatibility of the wheel and rail profile based on the vehicle characteristics and the operating condition. Zhang et.al [12] applied the partial rail profile expansion method to design wheel profile with Chinese $60 \mathrm{~kg} / \mathrm{m}$ rail. Shevtsov et al.[13-14] presented a numerical optimization technique based on rolling radius difference (RRD) of

wheelset to design the wheel profile. Shen et.al [15-17] developed a method, so-called "inverse methodology", for the design of railway wheel profile involving contact angle and rail profile information. A similar approach was proposed by Jahed et al.[18] wherein the RRD function was also used for the design of railway wheel profiles. Polach [19] proposed a new wheel tread profile design method which was considering the target conicity and wide tread wear spreading. Ignesti et.al [20] presented two profiles designed with the objective of improving stability 
behavior and minimising the wear. Cui et.al [21-22] used a wheel profile design method for EMU train with the considerations of the suspension parameters and wear performance.

The above-mentioned studies have summarized some fundamental studies and field experience on the development of wheel profile, and clear development of typical wheel profile issues are introduced, including the reasons why a wheel profile is the best alternative solution to satisfy the requirements of different operating conditions. However, most of current wheel profile optimization methods are aimed at vehicle dynamic behavior on tangent track. Only a few articles considering flange wear are simple verification of the wheel flange wear after optimization, and the optimization design goal of combining running behavior on tangent line and flange wear has not been given directly.

According to the rapid development of the EMU trains in last two decade, the increasing vehicle speed and carrying capability bring new challenges for the design of wheel profiles. The CRH1 train is one of the main commuter trains in China. The CRH1 trains are running on the majority of the normal speed lines and high speed lines. The quality of high-speed lines is much better than the normal speed lines. The LMA profile is a wheel profile which is used on high-speed Locomotive and car in China, but this wheel profile is not able to achieve the requirements of the suspension of the CRH1 train, and the serious lateral vibration occurs when it operates at highspeed. The alternative wheel profile, LMD, was designed to improve the stability of the wheelset but it can cause the wear on the wheel flange/rail gauge corner. The unexpected wear can definitely increase the cost of wheel/rail maintenance and also has impacts on the vehicle dynamics in some sections.

In order to design an optimal wheel profile for the CRH1 train, the performance of LMA and LMD was comparatively evaluated, including the wheel/rail contact geometry characters, the wheel/rail contact mechanical property and the vehicle dynamic behavior. Then a multi-objective optimization method was developed to reduce wear of wheel/rail and improve stability of wheelset. Finally, a new wheel profile was proposed in terms of the optimization method and the behavior of the CRH1 train was discussed.

\section{Performances of LMA and LMD}

LMA and LMD are the two typical wheel profiles which are used for CRH1 trains. Attempting to obtain a better profile, the performance of LMA and LMD was carefully analysed to expose their advantages. In the analysis, the Chinese $60 \mathrm{~kg} / \mathrm{m}$ rail (CN60) with $1353 \mathrm{~mm}$ rail gauge and 1/40 rail cant was used. The back to back distance of the wheel is set to $1435 \mathrm{~mm}$. 
The design of CRH1 bogie is shown in Fig.1 and the main parameters of the CRH1 train are listed in Table 1. In the optimization progress, only a trailer car is selected and applied, the evaluation is assumed to ignore the influence of train formation.

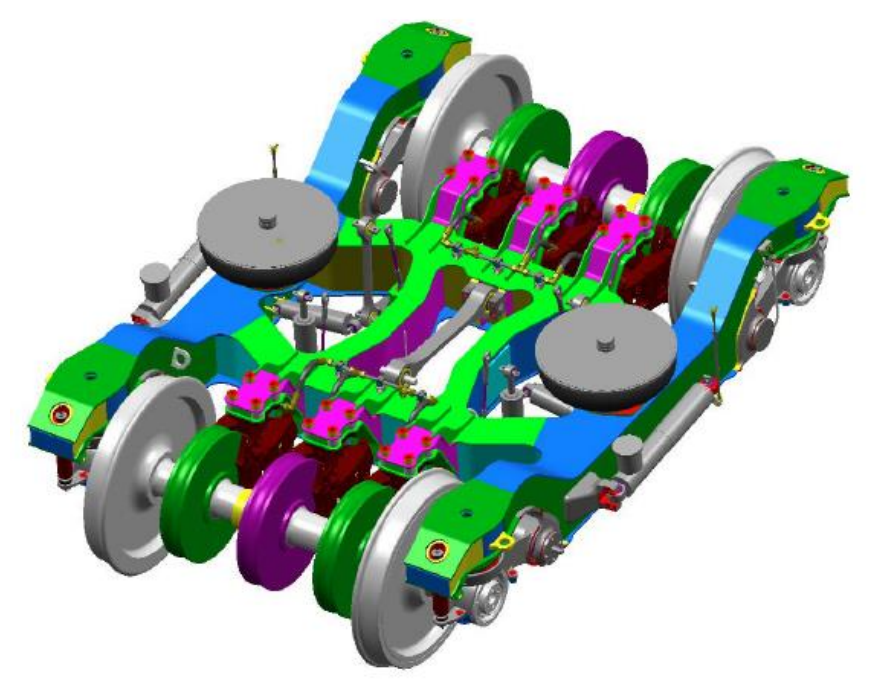

Fig.1 The bogie of CRH1 train

Table 1

Main parameters of the CRH1 train.

\begin{tabular}{lll}
\hline Mass of the coach & 40560 & $\mathrm{~kg}$ \\
Mass of the bogie & 1432 & $\mathrm{~kg}$ \\
Mass of the wheelset & 1621 & $\mathrm{~kg}$ \\
Primary suspension, longitudinal stiffness & $2.4 \mathrm{E} 7$ & $\mathrm{~N} / \mathrm{m}$ \\
Primary suspension, lateral stiffness & $6.0 \mathrm{E} 6$ & $\mathrm{~N} / \mathrm{m}$ \\
Primary suspension, vertical stiffness & $7.965 \mathrm{E} 5$ & $\mathrm{~N} / \mathrm{m}$ \\
Primary suspension, vertical damper & $1.1 \mathrm{E} 4$ & $\mathrm{~N} . \mathrm{s} / \mathrm{m}$ \\
Primary suspension, Bush vertical stiffness & $3.0 \mathrm{E} 6$ & $\mathrm{~N} / \mathrm{m}$ \\
Secondary suspension, longitudinal stiffness & $2.57 \mathrm{E} 5$ & $\mathrm{~N} / \mathrm{m}$ \\
Secondary suspension, lateral stiffness & $2.57 \mathrm{E} 5$ & $\mathrm{~N} / \mathrm{m}$ \\
Secondary suspension, vertical stiffness & $2.55 \mathrm{E} 5$ & $\mathrm{~N} / \mathrm{m}$ \\
Secondary suspension, anti-yaw damper & Nonlinear, see Table 2 \\
Secondary suspension, lateral damper & $3.7 \mathrm{E} 4$ & $\mathrm{~N} . \mathrm{s} / \mathrm{m}$ \\
Secondary suspension, Bush lateral stiffness & $3.0 \mathrm{E} 7$ & $\mathrm{~N} / \mathrm{m}$ \\
\hline
\end{tabular}

Table 2 
Nonlinear characteristics of anti-yaw damping of the secondary suspension stage.

\begin{tabular}{lllll}
\hline Velocity (m/s) & 0.01 & 0.04 & 0.1 & 0.2 \\
Force $(\mathrm{N})$ & 4600 & 12000 & 13500 & 15500 \\
\hline
\end{tabular}
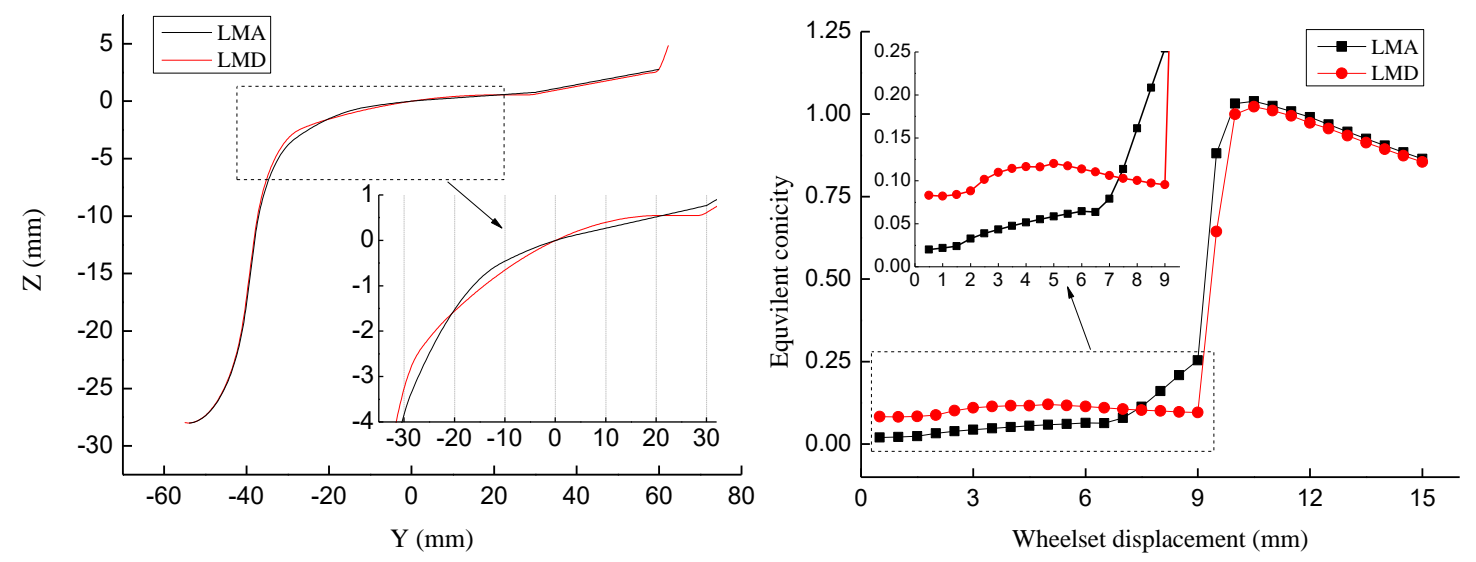

Fig.2 Wheel profiles LMA and LMD Fig.3 Wheel tread conicities for LMA and LMD wheels on CN60 rail.

First of all, it worth to discuss the geometry of the wheel profiles. LMA wheel profile was in use prior to the LMD profile designed. The geometry of the two proposed wheel profiles are shown in Fig.2. They were designed to have the same flange shape and rim width, and obvious difference can be found in the region of wheel tread and flange root. The slope of LMD is slightly higher than that of LMA and it is potential to increase the wheel tread conicity, as shown in Fig.3.

The wheel tread conicity is considered as the indirect index of the dynamic behavior of the wheelset. The wheel tread conicity can be classified into 3 regions. The first one is related to the tread contact within $3 \mathrm{~mm}$ lateral displacement of wheelset. This region is responsible for motion on a straight track. The second region corresponds to $3 \sim 6 \mathrm{~mm}$ lateral displacement of wheelset, and this region is design to take effect of the curves with a large radius. The third one corresponds to the negotiation on sharp curve with the flange contact beyond $6 \mathrm{~mm}$ wheelset displacement.

In Fig.3, it can be seen that the wheel tread conicity of LMD profile is much higher than that of LMA profile within $7 \mathrm{~mm}$ lateral displacement of wheelset. For the CRH1 train, the raising tread conicity in the region of 8$10 \mathrm{~mm}$ wheelset displacement can significantly increase the guiding force of the wheelset to improve the vehicle stability on straight track and curve line with a large radius. However, the transitional region of the LMD tread conicity from wheel tread to flange is not smooth enough as expected. In this manner, when a vehicle is passing the 
sharp curve, the guiding force of wheelset will be not enough within $9 \mathrm{~mm}$ wheelset displacement, then the lateral displacement of wheelset should be increased exceed $9 \mathrm{~mm}$. In this case, the wheel flange will certainly contact with rail gauge corner to steer the wheelset and then cause wheel flange/rail gauge corner wear.
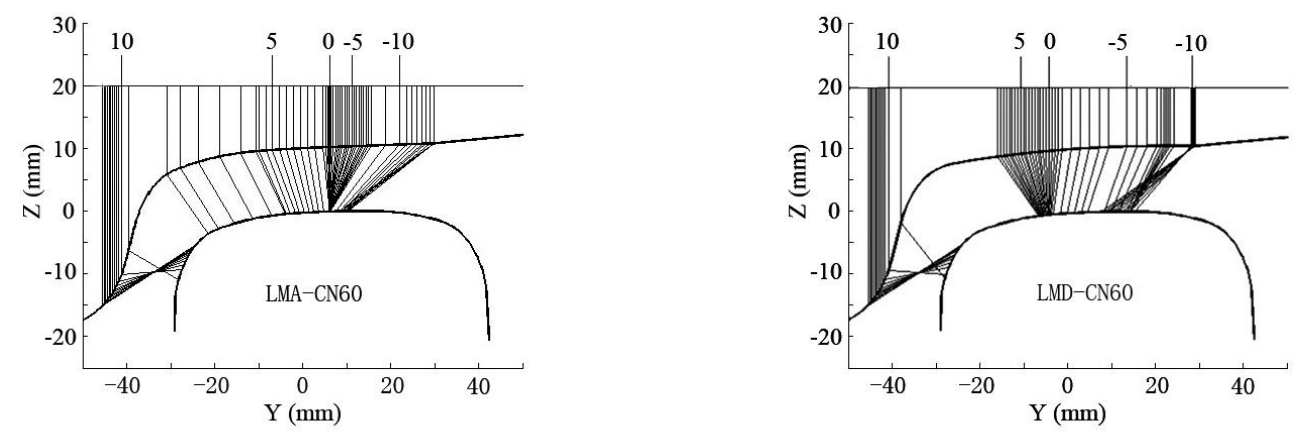

Fig.4 Position of contact points on wheel and rail depending on lateral displacement of wheelset

The contact points of the LMA and LMD wheel profiles on CN60 rail are shown in Fig.4. The lines between the wheel and rail represent the corresponding contact points and the value of corresponding lateral displacements of wheelset are shown in above the wheel profile. The contact points of LMD profile are more dispersed than that of LMA profile. Within 9mm lateral displacement of wheelset, the contact points of LMD profile normally concentrate around the middle of the wheel tread. When a vehicle passes a sharp curve, the LMD profile is not able to provide enough guiding force in contact area, while the displacement of wheelset continuously keep increasing until the wheel flange meets the rail.

The results of dynamic simulations of the CRH1 vehicle with LMA and LMD wheel profiles are shown in Fig.5 to Fig.11. The CRH1 train model was built in a multi-body dynamic software, SIMPACK. The critical speed of the vehicle with the two profiles is set to $374 \mathrm{~km} / \mathrm{h}$ in LMA and $373 \mathrm{~km} / \mathrm{h}$ in LMD, which are fast enough to meet the requirement of $200 \mathrm{~km} / \mathrm{h}$ for the operation speed. The difference of the wheel geometry leads to different vehicle dynamic behaviors between LMA and LMD. Fig. 5 shows the lateral acceleration of wheelset and car body when the vehicle running on a tangent line with measured rail irregularity in Chinese high-speed rail. In Fig.5, it is clear that the lateral acceleration of wheelset and car body using LMD profile is much smaller than that of LMA profile. It is obvious that the LMD profile can slightly increase the stability of the CRH1 vehicle. 


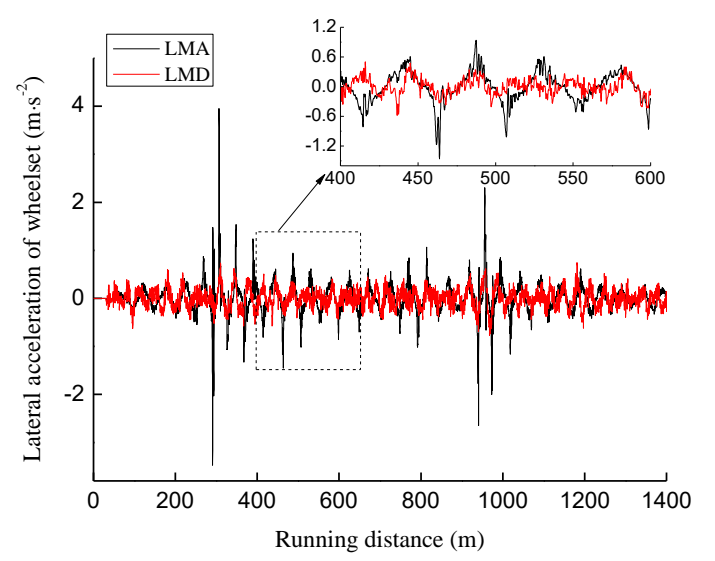

(a) Wheelset

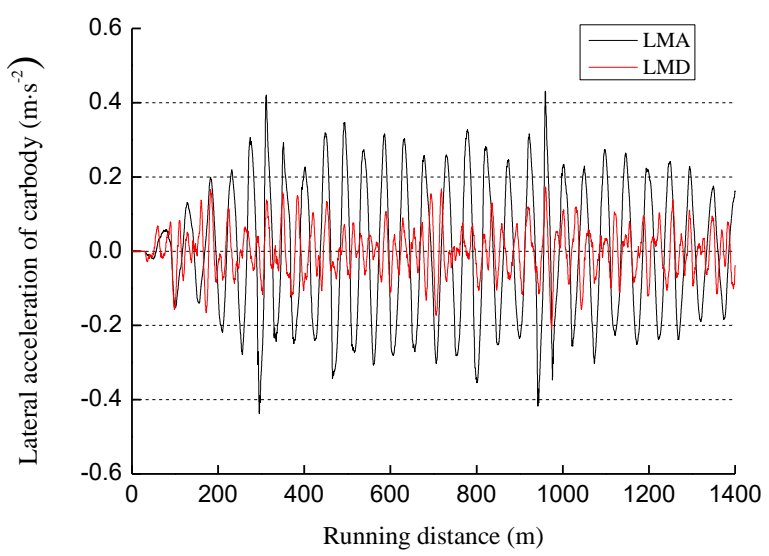

(b) Car body

Fig.5 The lateral acceleration

The ride indexes of the vehicle at different speeds are simulated and calculated using Sperling's filter [23] as shown in Fig.6. The lateral ride indexes of the vehicle with two different profiles can both increase with the speed increasing. However, the growth of LMD profile is slower than of LMA profile. When the vehicle speed exceeds $140 \mathrm{~km} / \mathrm{h}$, the ride indexes of LMD profile start to provide smaller value than that of LMA profile, which means the LMD profile is sufficient to provide better ride comfort to the CRH1 vehicle on tangent track.

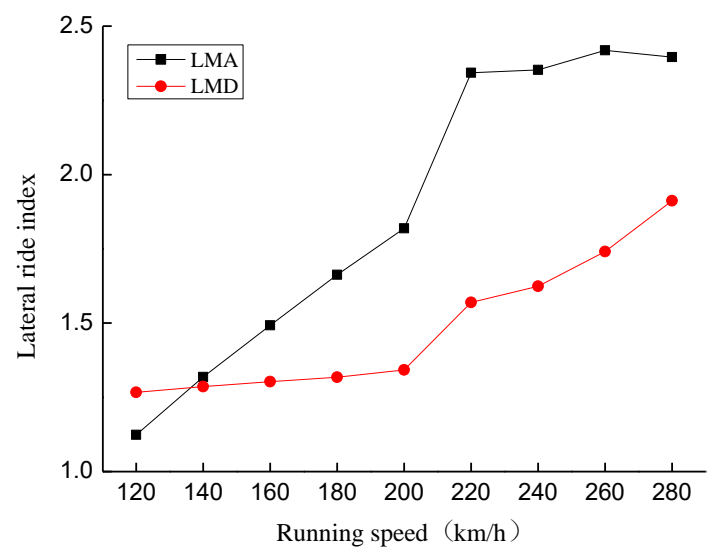

Fig.6 Lateral ride index

Most of the high-speed lines are composed of the tangent track or curved track with the radius greater than $6000 \mathrm{~m}$. In recent study, a curved section which consists of $2000 \mathrm{~m}$ is used for a sample track in dynamic simulation. The first section of track line was designed with a tangent part of $320 \mathrm{~m}$, a transition part of $200 \mathrm{~m}$, a curved part of $1000 \mathrm{~m}$ and a radius of $7000 \mathrm{~m}$, and then extended by connecting to a transition part of $200 \mathrm{~m}$ and a straight part of 
$280 \mathrm{~m}$ in the rest of track line, as shown in Fig.7 curve 1. The super elevation is $100 \mathrm{~mm}$. Fig.7 gives the dynamic behavior when the CRH1 vehicle passing that track at a speed of $200 \mathrm{~km} / \mathrm{h}$. The vehicle using LMA wheels is unstable on transition part of the track. The lateral displacement of LMD wheelset is smaller than that of LMA wheelset on the curved track due to the LMD has the larger wheel tread conicity which can provide larger guiding force.
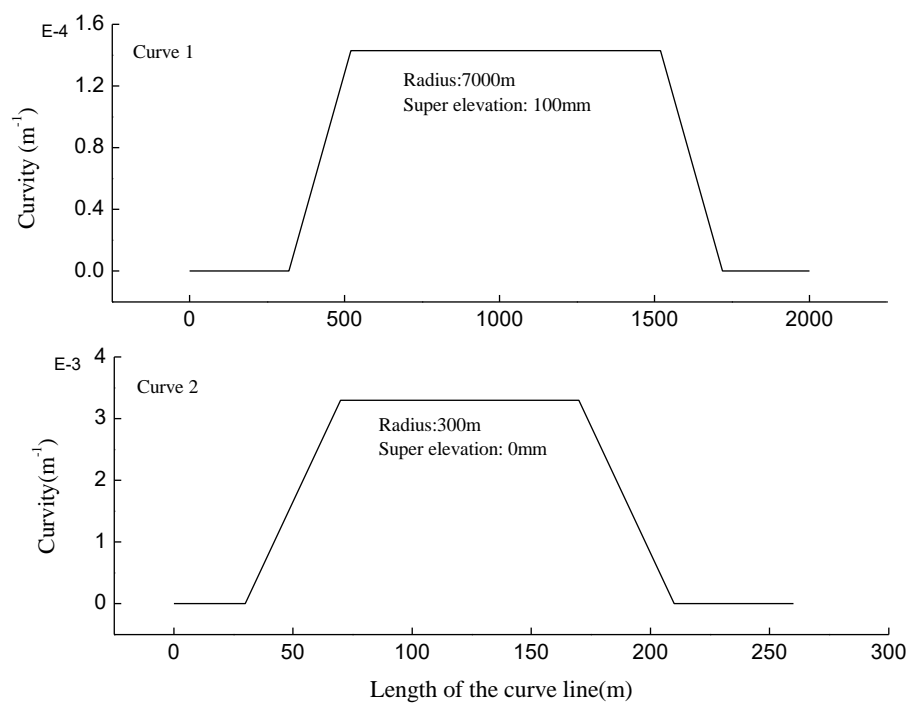

Fig.7 Parameters of the curve lines

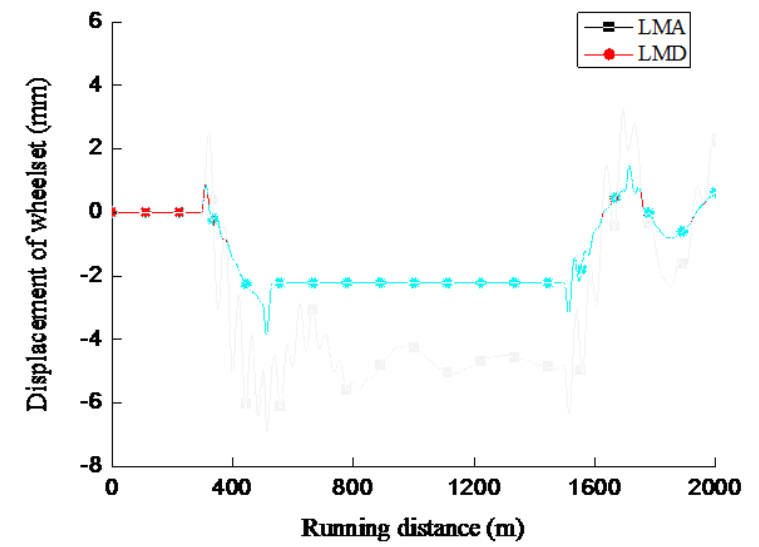

(a)Lateral displacement of the wheelset

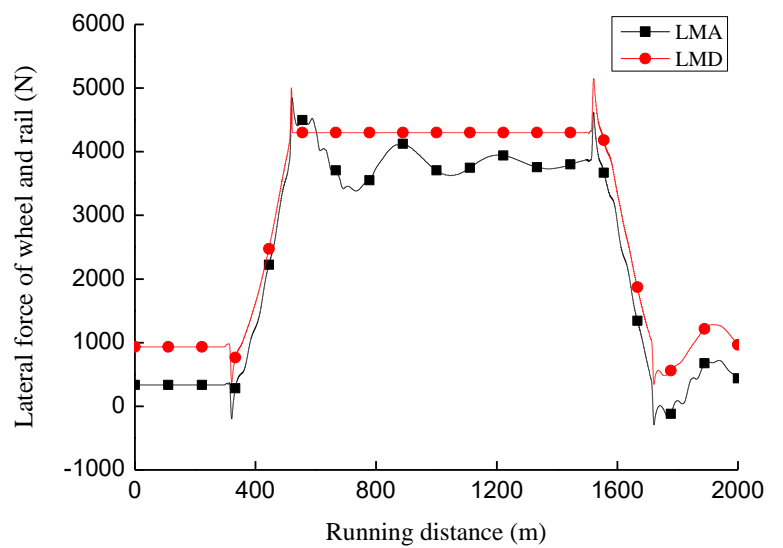

(b)Lateral force of wheel and rail

Fig.8 Curve negotiation performance with large radius

The curving performance, when vehicle negotiates a narrow curve, is also simulated as shown in Fig.9. The curve is set to relate to a realistic line in Shanghai as shown in Fig.7 curve 2, 30m tangent track, 40m transition track, then a curved part of $100 \mathrm{~m}$ with a radius of $300 \mathrm{~m}$, followed by $40 \mathrm{~m}$ transition line and $50 \mathrm{~m}$ straight line. There is 
no super elevation on this curve. The train running speed is set to $9 \mathrm{~km} / \mathrm{h}$. When the CRH1 vehicle negotiates on the narrow curve, the lateral displacements of the LMA and LMD wheelsets are both around 9.5mm, which can cause the contact between wheel flange and the rail and result in significant wear of wheel flange/rail gauge corner. Actually, when a vehicle passes this curve, the large lateral displacements of wheelset and angle of attack can bring about two-point-contact [24]. Additionally, the lateral force of LMD wheel is larger than that of LMA wheel as shown in Fig.9(b), which indicates that a larger wheel flange/rail gauge corner wear occurred by using LMD wheel.

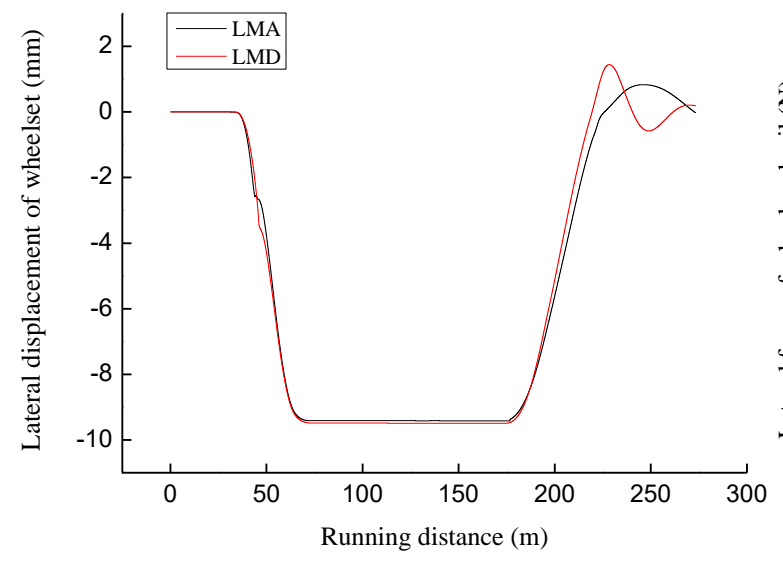

(a) Lateral displacement of wheelset

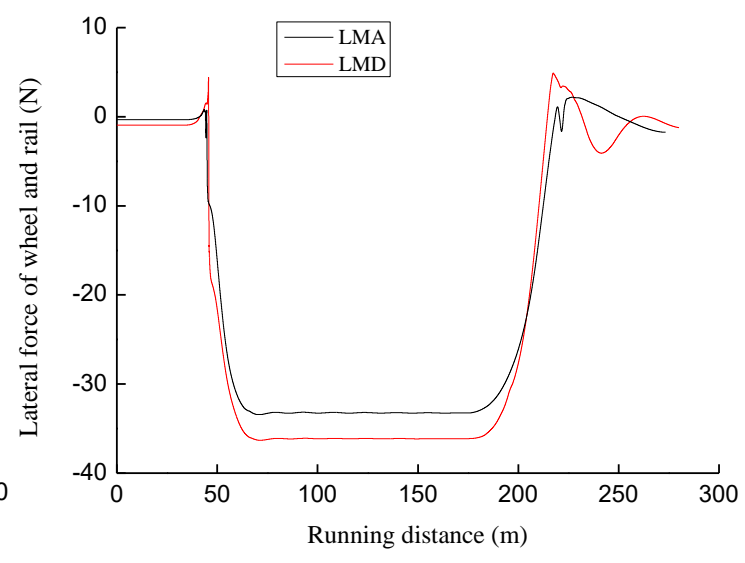

(b) Lateral force of wheel and rail

Fig.9 Curve negotiation performance with narrow radius

\section{Optimization Problem Definition}

\subsection{Design variables}

In the study of the wheel optimization, the profile can normally be described in two ways. One derives some relationship to get the mathematical expression of the wheel profile [11,12,17], and the other applies the interpolating fitting curve to represent the wheel profile $[13,14,18,21]$. In this study, 15 points on the profile have been selected between flange and filed side to represent the wheel profile as shown in Fig 10. The nodes on the surface of the wheel profile express as the uniform distribution on the wheel profile from $-40 \mathrm{~mm}$ to $30 \mathrm{~mm}$. Point $A$ is on the position of the rolling circle in name at coordinates of $(0,0)$. Point $B$ is on the wheel flange and the lateral distance to point A is $40 \mathrm{~mm}$. Point $A$ and $B$ have been fixed to ensure the profile have the same size of wheel flange and wheel diameter. The lateral coordinates of the moving nodes are set to be constant, and the vertical coordinates are considered as variable values. The wheel profile in the optimization region can be generated through fitting the 
constant and variable nodes by using cubic spline function. The original wheel flange curve can be directly connected to the optimization region, and a straight line which has the same slope as the last nodes $C$ is also connected, then a complete wheel profile can be created and expressed by the vertical coordinates $z_{0}, z_{1}, \ldots, z_{14}$ as $f(\mathbf{z})$. There, $\mathbf{z}=\left[z_{1}, z_{2}, \ldots, z_{8}, z_{10}, \ldots, z_{14}\right]$ is the design variable.

To ensure wheel flange and straight line smoothly, some boundary conditions are set at the start point $B$ and end point $C$ as follow

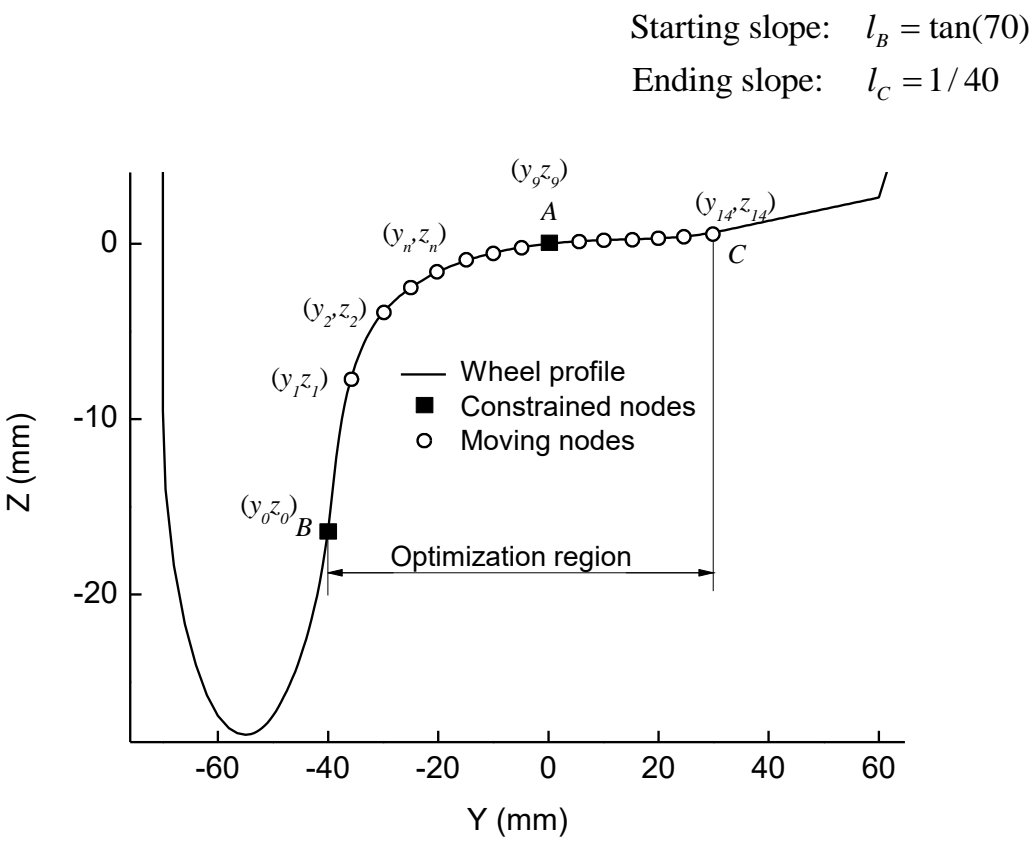

Fig.10 The expression of the wheel profile

\subsection{Objective function}

Based on the above study and the actual operation of the CRH1 train, the LMA and LMD wheels both of them have advantages and disadvantages. In recent study, the main objective is to create an optimal wheel profile which can coordinate the trade-off between ride comfort (better in LMD profile) and wheel flange/rail gauge corner wear (less in LMA profile).

The most direct goal of wheel tread design is, of course, to achieve the optimal running stability and ride comfort of the vehicle and the lowest wear index of the flange and tread, but these parameters cannot be obtained at the same time. The dynamic behaviors of vehicle running on tangent or curved track are even contradictory. In addition, the relationship between these performance indexes and wheel profile cannot be directly established by 
mathematical expressions, but must be correlated with each other by numerical methods, which makes it difficult to establish objective functions and select optimization methods.

In order to simplify the calculation process, the lateral acceleration of the car body is selected to represent the ride comfort index in tangent track and large curved track, the lateral force of wheel and rail and angle of attack are chosen to indicate the wheel flange/rail gauge corner wear. Therefore, the target functions are written as

$$
\text { Minimize: } \begin{gathered}
\left\{\begin{array}{c}
f_{1}(\mathbf{z})=\max \left(\left|\varphi_{i}\right|\right) \\
f_{2}(\mathbf{z})=\max \left(\left|Q_{i, j}\right|\right) \\
f_{3}(\mathbf{z})=\operatorname{Rms}(A)
\end{array}\right. \\
(i=1,2,3,4 ; j=l, r)
\end{gathered}
$$

where $f_{1}$ is the maximum angle of attack of the four wheelsets in the vehicle. $\varphi_{i}$ is the angle of attack of the $i^{\text {th }}$ wheelset. $f_{2}$ is the maximum lateral force of $j$ side wheel/rail on the $i^{\text {th }}$ wheelset. $f_{3}$ is the root mean square value of car body acceleration $A$. The measurement point of the acceleration is one of the marker points of air springs on the car body. The RMS value is calculated over $100 \mathrm{~m}$ distance with a step of $10 \mathrm{~m}$.

In order to obtain an integrated objective function, the amplification coefficients are employed to change the values of the three targets to the same magnitude. In addition, the weighted factors are used to consider the importance of each objective. So the objective function can be written as

$$
f(\mathbf{z})=10000 w_{1} f_{1}+w_{2} f_{2}+100 w_{3} f_{3}
$$

\subsection{Design constraints}

The design variables must meet the requirement of the monotonicity of the wheel profile curve. Hence, the constraint equation is employed as

$$
G_{k}=\frac{z_{k}-z_{k-1}}{y_{k}-y_{k-1}}>0, \quad k=1,2, \ldots, 14
$$

In optimization process, the vehicle dynamic behaviors are calculated to ensure the generate wheel profile meet the requirement of derailment security, denotes by

$$
g_{1}=\max \left(\left|\frac{Q_{i, j}}{P_{i, j}}\right|\right)<1
$$

and 


$$
g_{2}=\max \left(\left|\frac{P_{i, l}-P_{i, r}}{P_{0}}\right|\right)<0.6
$$

In Eq.(5) and Eq.(6), $P$ is the vertical force of wheel and rail. $Q$ is the lateral force of wheel and rail. $P_{0}$ is half of the axle load.

\section{Optimization Algorithms}

According to Eqs. (1)-(9), the optimization method can be described by

$$
\begin{aligned}
& \text { Minimum: } f\left(z_{1}, z_{2}, \ldots, z_{14}\right)=f(\mathbf{z}), \mathbf{v} \in \mathbf{R}^{N} \\
& \text { Subject to: } \mathrm{G}_{k}>0, g_{1}<1, g_{2}<0.6
\end{aligned}
$$

It is clear that all the functions of this method have no analytical expressions and can be obtained through Simpack computational package, and it can be seen as the limitation of using the conventional optimization method

In this section, the particle swarm optimization (PSO) $[25,26]$ is used to solve the problem. In order to improve calculation efficiency and ensure the better convergence properties of the algorithm, the time-varying acceleration coefficients are given as:

$$
c_{1 t}=\alpha \frac{M-t}{M}+c_{1}, c_{2 t}=\alpha \frac{t}{M}+c_{2}
$$

where $c_{1}, c_{2}, \alpha$ are constants. $M$ is the maximum number of allowable iterations. $t$ is the current iteration number. $c_{1 t}$ and $c_{2 t}$ are the current acceleration coefficients.

The iterative formula can be written as

$$
\begin{aligned}
& v_{n d}(t+1)=\chi\left[v_{n d}(t)+c_{1 t} r_{1}\left(p_{n d}(t)-x_{n d}(t)\right)+c_{2 t} r_{2}\left(p_{g d}(t)-x_{n d}(t)\right)\right] \\
& z_{n d}(t+1)=z_{n d}(t)+v_{n d}(t+1)
\end{aligned}
$$

In Eq.(9), $n=1,2, \ldots, N$ and $N$ is the number of the wheel profiles in an algorithm called population dimension. $d=1,2, \ldots, D$ and $D$ is the dimension of variables vector space. In this study, $D=13, v_{n d}$ is termed the velocity of each particle, according to its own flying experience and the flying experience of the other particles in the search space. $z_{n d}$ is the position of each particle and the position of each particle is a potential solution. $p_{n d}$ is the best position of each particle, and $p_{g d}$ is the fittest particle found so far at the time $t . r_{1}$ and $r_{2}$ are the pseudo random number independent of each other and submit to a uniform distribution in $[0,1] . \quad \chi$ is the compress factor and expressed as 


$$
\chi=\frac{2}{\left|2-C-\sqrt{C^{2}-4 C}\right|}, C=c_{1 t}+c_{2 t}, C>4
$$

The typical structure of the PSO method using on a wheel profile optimization is shown in Fig.11 In this study, $c_{1}=2.5, c_{2}=2 ; \alpha=1.5, M=150, N=5$. The population dimension $N$ and the maximum number of allowable iterations $M$ dictates the computing time and accuracy. Of course, the parallel programs can be used to run Simpack model with $N$ wheel profiles at the same time. There are 3 computing cases in Simpack, tangent track, large curved track and sharply curved track. The parameters of each case are set as chapter 2 . The target function $f_{l}$ and $f_{2}$ are only related to the sharply curved track, and $f_{3}$ is decided by tangent track and large curved track.Therefore, the weighted factors are set as $w_{1}=w_{2}=0.3$ and $w_{3}=0.4$ based on experience.

In the optimization progress, the termination conditions are set as

$$
\begin{aligned}
& \min \left(f_{n}^{t-1}\right)-\min \left(f_{n}^{t}\right)<\varepsilon \\
& t=M
\end{aligned}
$$

where, $\varepsilon$ is the allowable tolerance.

\section{Results and Discussion}

The optimization procedure is shown in Fig.11 has been implemented using MATLAB software. The first work in progress of optimization is set the parameters, $c 1, c 2, N, M$, which used in PSO method. Then $N$ sets of $\mathbf{z}$ are produced in random and $N$ wheel profiles are generated through connecting each sets of $\mathbf{z}$ using cubic spline function meeting Eq.(1). Send the profiles into vehicle dynamic model established in Simpack and do the simulation. If the results meet Eq.(5) and Eq.(6), then update the parameters $p_{n d}$ and $p_{g d}$ and judgment the termination conditions Eq.(11). If meeting Eq.(11), the optimization break and obtain the minimum value of $f(\mathbf{z})$ and the corresponding $\mathbf{z}$. Then the optimization profile can be obtained by connect each point using cubic spline function based $\mathbf{z}$. If the results don't meet Eq.(5), Eq.(6) or Eq.(11), the $v_{n}(t+1)$ and $z_{n}(t+1)$ will be gotten according to Eq.(9) and others $\mathrm{N}$ wheel profiles will be generated. The whole optimization progress is in a close loop until obtain a optimized profile.

The result OPT profile is shown in Fig.12 compared with LMA and LMD profiles. The OPT profile has the similar shape with the LMA and LMD profiles close to the rolling circle in name, but also have a distinct difference on the flange root section in comparison to two previous profiles. This is because the LMD profile has outstanding behavior on tangent track but it is not able to provide sufficient guide force on a curved track. From Fig.13, where 
the equivalent conicity is shown, it is evident that the OPT profile has the similar values of equivalent conicity within $7 \mathrm{~mm}$ wheelset later displacement which ensures the outstanding behavior of wheels on the tangent track. Meanwhile, the conicity in the range from $7 \mathrm{~mm}$ to $9 \mathrm{~mm}$ wheelset displacement increases gradually, which can provide a better curving performance compared with the conicity status of LMD profile in this zone.

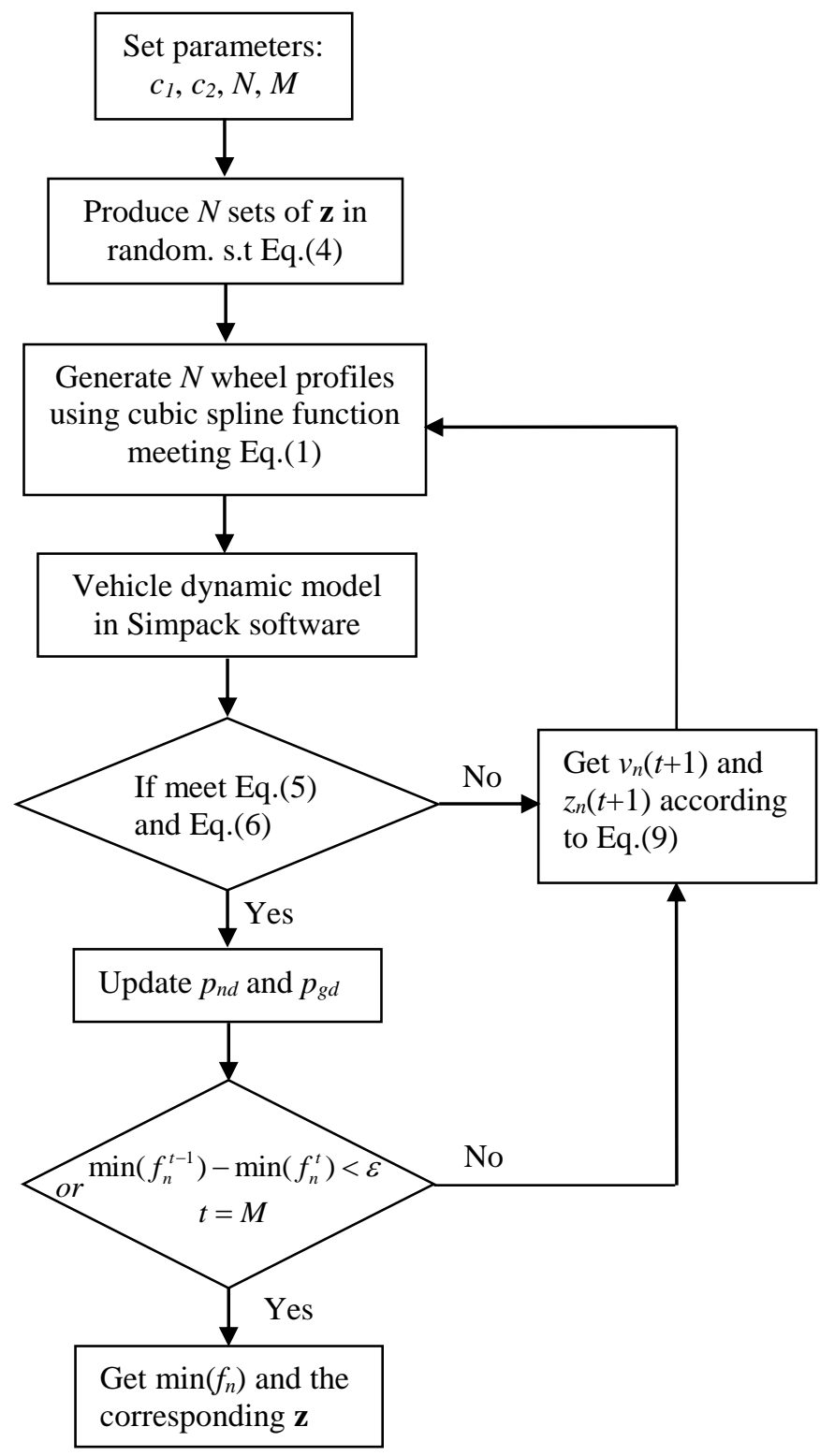

Fig.11 The follow chart of optimization 

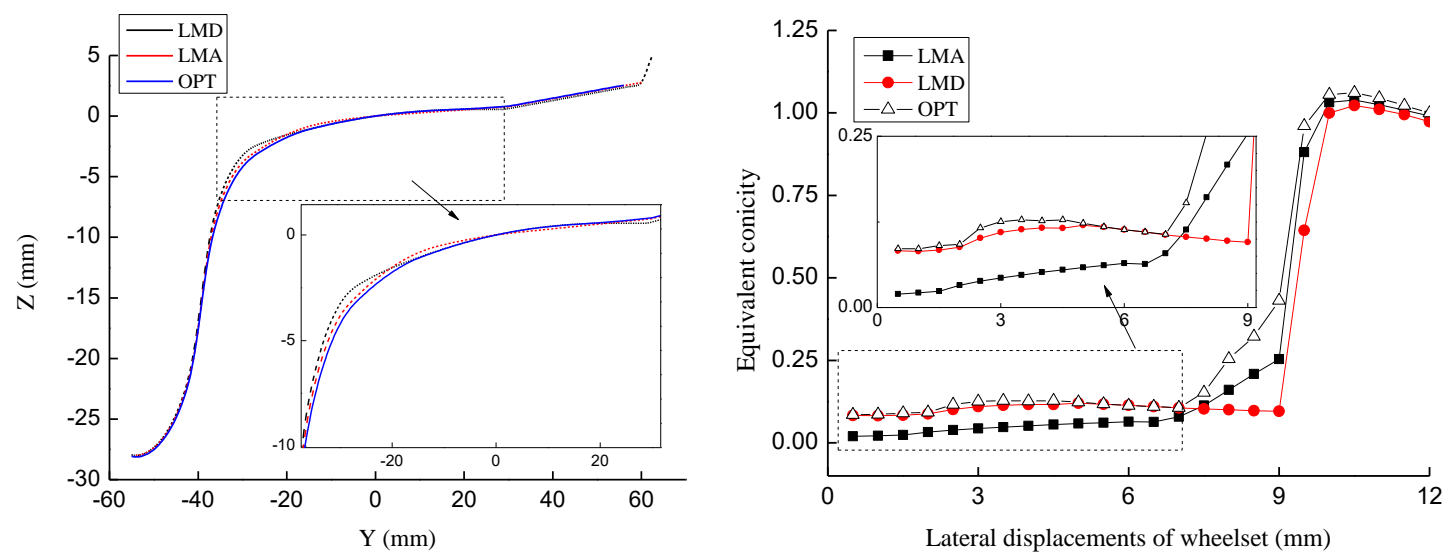

Fig.12 Wheel profiles LMD, LMA and OPT

Fig.13 Equivalent conicity of wheel profiles LMA, LMD and OPT

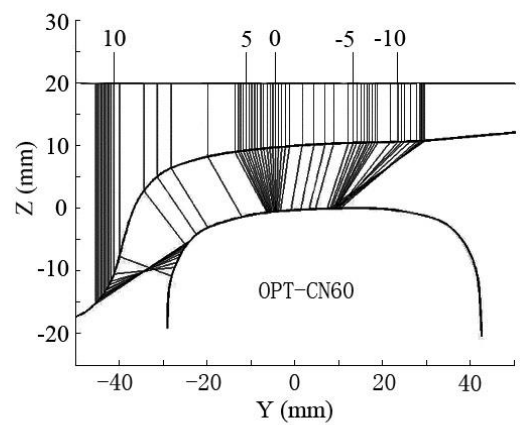

Fig.14 Position of contact points on wheel and rail

The distribution of contact points versus lateral displacement of the wheelset was calculated and shown in Fig.14. When the displacement of wheelset beyond $7 \mathrm{~mm}$, the contact points will pass through the wheel flange root gradually instead of jumping from wheel tread to the wheel flange as LMD profile. This contact characteristics have benefits for improving curving performance and reducing wheel flange wear.

Fig. 15 shows the displacement of the wheelset when the vehicle runs on a tangent track and passes a lateral sinusoidal irregularity excitation at a speed of $300 \mathrm{~km} / \mathrm{h}$. It can be seen that the vehicle with LMA profile is instable when passing the irregularity excitation. The OPT and LMD profiles both can ensure the vehicle passing the excitation smoothly. According to the result of the simulation, the OPT profile can provide a higher critical speed of $396 \mathrm{~km} / \mathrm{h}$, than that using LMD profile. The lateral ride index of the vehicle with OPT profile is higher than LMD profile when the vehicle running speed under $220 \mathrm{~km} / \mathrm{h}$. When the speed exceeds $220 \mathrm{~km} / \mathrm{h}$, the lateral ride index of OPT profile is reduce to be smaller than LMD profile as shown in Fig.16. 


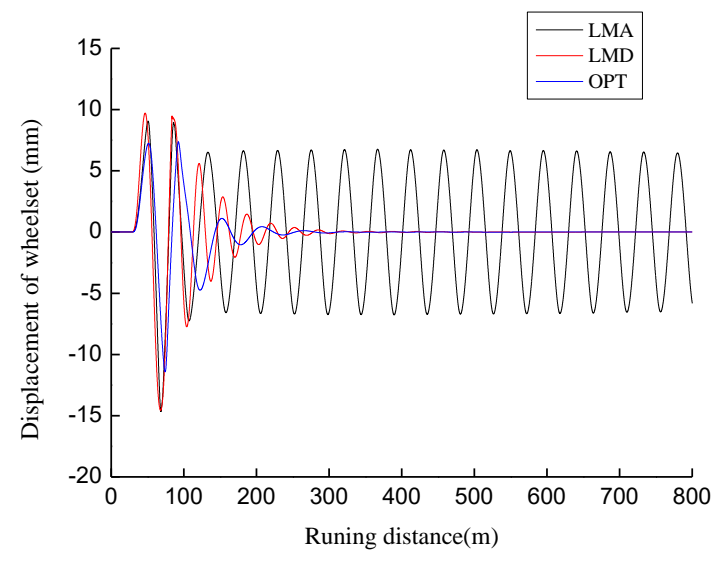

Fig.15 Displacement of the wheelset

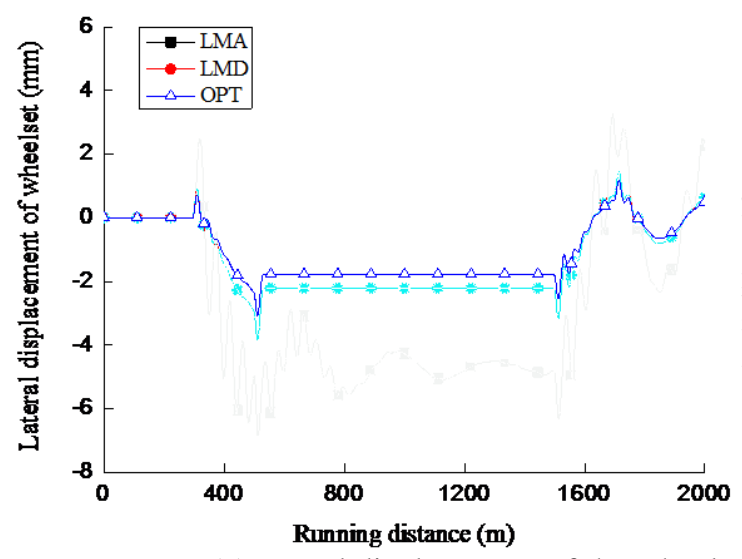

(a)Lateral displacement of the wheelset

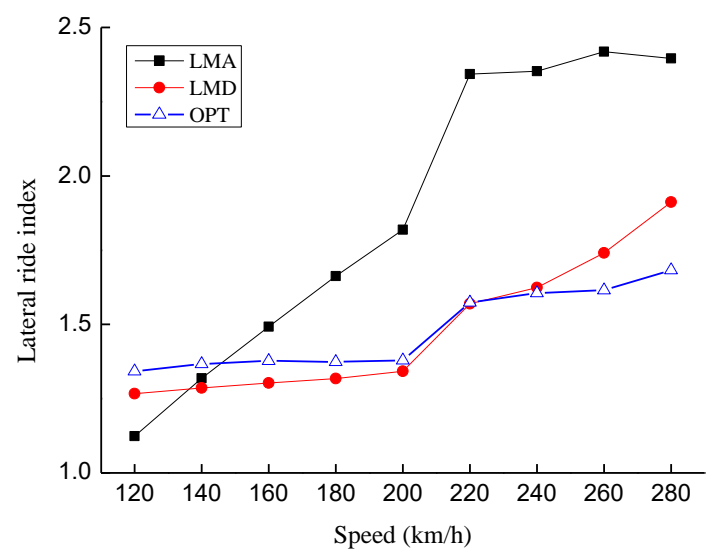

Fig.16 Lateral ride index

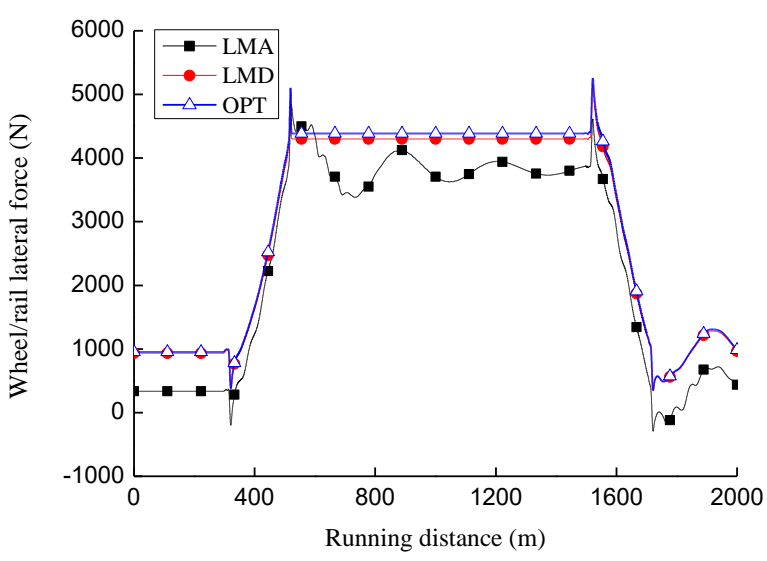

(b)Lateral force of wheel and rail

Fig.17 Curve negotiation performance with large radius

The curve negotiation performance of OPT profile with radius of $7000 \mathrm{~m}$ was calculated and are shown in Fig.17 compared with LMA and LMD profiles. It can be seen that the lateral displacement of the wheelset using OPT profile is the lowest one and the lateral force of wheel and rail is the highest in those three profiles due to the high conicity of OPT profile. When a vehicle passes the narrow radius curve, shown in Fig.18, the lateral displacement of the wheelset on OPT profiles is obviously lower than the other profile, which can reduce the lateral force between wheel flange and rail gauge corner. 


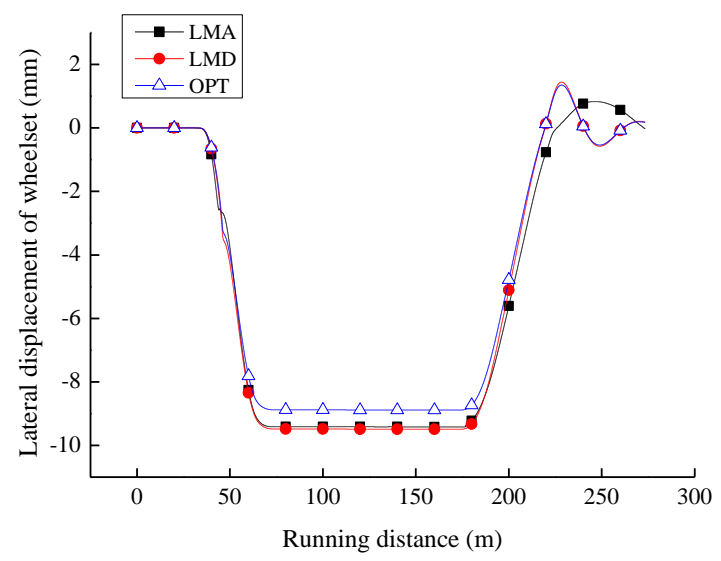

(a) Lateral displacement of wheelset

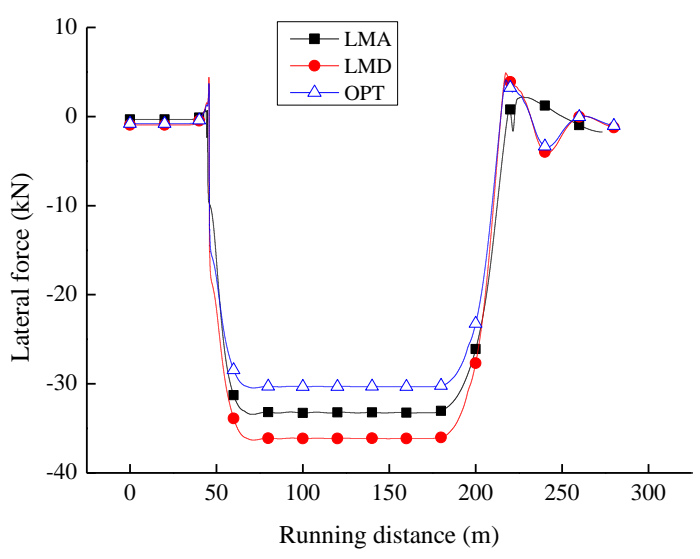

(b) Lateral force of wheel and rail

Fig.18 Curve negotiation performance with narrow radius

To compare the vehicle dynamic behavior of the three profiles, the curve negotiation performance with different radiuses were calculated as shown in Fig.19. It can be seen that the lateral displacement of wheelset and wheel/rail lateral force is gradually increased with the curve radius decreases. When the curve radius is over $3000 \mathrm{~m}$, the lateral displacement and force are changed slowly. Once the curve radius smaller than $3000 \mathrm{~m}$, the wheel flange will contact the rail and lead to the lateral displacement of wheelset and wheel/rail lateral force increase rapidly. According to OPT profile can employ the root of the wheel flange to translate the contact point from wheel tread to wheel flange, the displacement and wheel/rail force becomes smaller than LMD profile at the same curve radius.
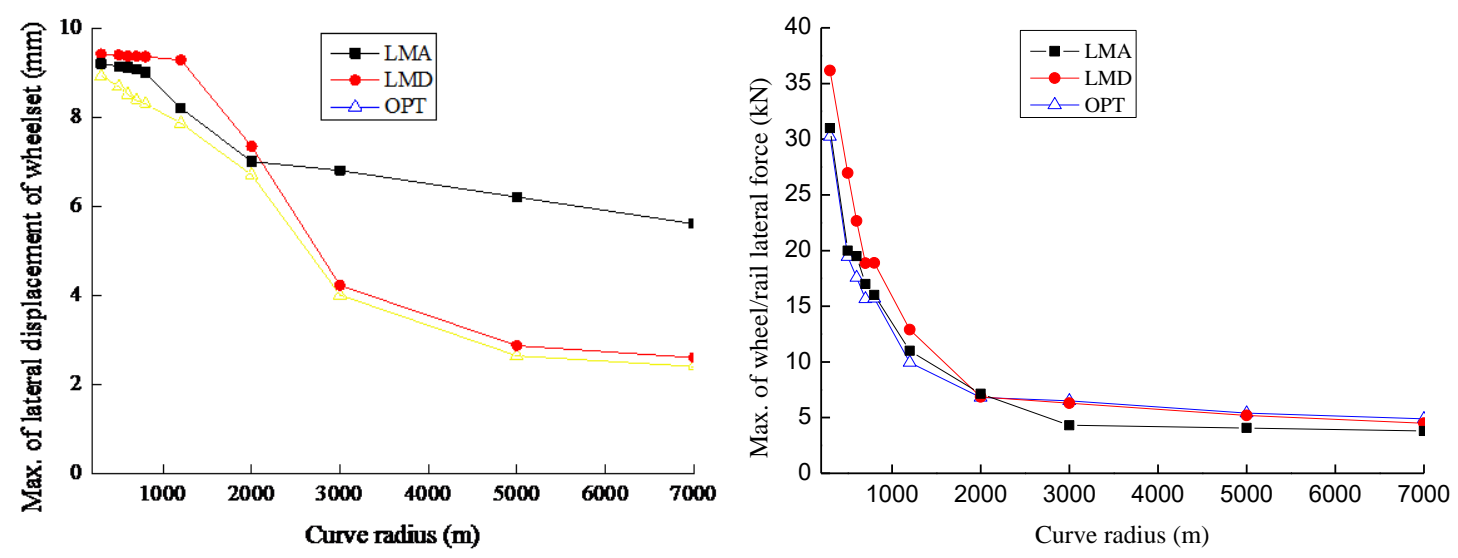

Fig.19 Curve negotiation performance with different radiuses

\section{Conclusions}

In the present work, a comparative analysis of the performances of the LMA and LMD profiles on the CRH1 vehicle is given. Subsequently, a multi-objective optimization method is introduced for the design of wheel profiles 
to reduce the wheel flange/rail gauge corner wear on the premise of ensuring the dynamic behavior of the vehicle. A closed loop program is completed in Matlab software, in which the particle swarm optimization method is employed for seeking the optimum profile and the vehicle dynamic behavior is obtained by Simpack.

The procedure is applied to the design of a new profile for the CRH1 train in China. Comparative results obtained from a behavior simulation show that the optimized profile can reduce wheel/rail lateral force in the sharply curved track without loss dynamic behavior in a tangent and large curved track.

This method is not limited to the objective function used here, but can easily be altered to add other criteria based on the problems occur in practice. It can also be applied to design a rail profile to determine the amount of rail grinding.

However, the wear and instability of vehicle are not solely due to the wheel profiles, modification of wheel profiles is also not the simple means to solve these problems. To solve such complex problems, the factors affecting the wheel flange wear, vehicle dynamic behavior and maintenance costs should be considered overall. Actually, simultaneously with the wheel profile optimization, track and vehicle parameters modification and implement lubrication measures were also be taken into account in this project.

\section{Acknowledgements}

The present work has been supported by the National Natural Science Foundation of China (Nos.51605394 and U1434210), the Department of Education Foundation of Sichuan Province(No.16ZB0011), and the Key Research Project of Leshan City(No. 16ZDYJ0147).

\section{REFERENCE}

[1] Jin Xuesong, Shen Zhiyun. Development of rolling contact mechanics of wheel/rail systems[J].Advances in Mechanics, 2001, 31(1): 33-46.

[2] Zhang Shuguang, Gu Jianguo. Thinking and assumption of independent innovation of wheelset for highspeed cars in China[J]. Rolling stock, 2009, 47(3): 1-5.

[3] WICKENS A H. Dynamics of railway vehicles - from Stephenson to Carter[J]. Proc Inst Mech Eng Part F J Rail Rapid Transit, 1998, 212(3): 209-217.

[4] Heumann H., Zur Frage des Radreifen-umrisses. Organ für die Fortschritte des Eisenbahnwesens, v.89, n. 18, Sep. 1934, pp. 336-342. 
[5] Yang Guozhen. A preliminary study of wheel tread shape (Upper section)[J]. Rolling stock, 1978, 11: 1-6.

[6] Yang Guozhen. A preliminary study of wheel tread shape (Lower section )[J]. Rolling stock, 1978, 12: 1-17.

[7] Liu Xinming. Development of arc-shaped tread contour for locomotives and rolling stock[J]. Rolling stock, 2000, 38(2): 24-28.

[8] Eisaku Sato. The scientific design of wheel tread shape[J]. Foreign diesel locomotive, 2000, 3: 39-43.

[9] Haque I, Latimer D A, Law E H. Computer-aided wheel profile design for railway vehicles [J]. ASME Journal of Engineering for Industry, 1989, 111: 288-291.

[10] Sato Y. History study on designing Japanese rail profiles. Wear, 2005, 258(7-8): 1064-1070.

[11] Wu H M. Investigations of Wheel/rail Interaction on Wheel Flange Climb Derailment and Wheel/rail profile Compatibility[D]. The Graduate College of the Illinois Institute of Technology.2000.

[12] Zhang Jian, Wen Zefeng, Sun Liping, Jin Xuesong. Wheel profile design based on rail profile expansion method[J]. Chinese Journal of Mechanical Engineering, 2008, 44(3): 44-49.

[13] Shevtsov I Y, Markine V L, Esveld C. Optimal design of wheel profile for railway vehicles[J]. Wear, 2005(258): 1002-1030.

[14] Markine V.L, Shevtsov I.Y, Esveld C. An inverse shape design method for railway wheel profiles[J]. Struct Multidisc Optim, 2007, 33: 243-253.

[15] Shen G, Ayasse J B, Chollet H, et.al. Aunique design method for wheel profiles by considering the contact angle function[J]. Proceeding of the I MECH E, Part F J. Rail Rapid Transit, 2003 (217): 25-30.

[16] Shen Gang, Chollet H, Ye Zhisen. Study on wheel profile and contact analysis[J]. Journal of the China railway society, 2005, 27(4): 25-29.

[17] Shen Gang, Zhong Xiaobo. Inverse method for design of wheel profiles for railway vehicles. Journal of mechanical engineering, 2010, 16:41-47.

[18] Hamid Jahed, Behrooz Farshi, Mohammad A. Eshraghi, Asghar Nasr A numerical optimization technique for design of wheel profiles[J]. Wear, 2008(264): 1-10.

[19] Polach O. Wheel profile design for target conicity and wide tread wear spreading[J]. Wear, 2011, 271: 195201.

[20] Ignesti M, Innocenti A, Marini L, Meli E, Rindi A, Toni P. Wheel profile optimization on railway vehicles from the wear viewpoint. International Journal of Non-Linear Mechanics, 2013,53:41-54. 
[21] Cui Dabin, Li Li, Xuesong Jin. Optimal design of wheel profiles based on weighted wheel/rail gap [J]. Wear, 2011 271: 218-22.

[22] Cui D.B., Wang H.Y., Li L., Jin X.S. Optimal design of wheel profile for high-speed train. Proc IMechE Part F: J Rail and Rapid Transit. 2015, Vol. 229(3):248-261.

[23] CUI Dabin, LI Li, JIN Xuesong, LI Ling. Wheel-rail profiles matching design considering railway track parameters. Chinese Journal of Mechanical Engineering.2010, 23(4): 410-417.

[24] Dabin Cui, Weihua Zhang, Guangdong Tian, et.al. Designing the key parameters of EMU bogie to reduce side wear of rail. Wear, 2016, 366-367:49-59.

[25] Clerc M, Kennedy J. The particle swarm-explosion, stability, and convergence in a multidimensional complex space[J]. IEEE Transactions on Evolutionary Computation, 2002, 6(1): 58-73.

[26] Ratnaweera A, Halgamuge S. Self-organizing hierarchical particle swarm optimizer with time-varying acceleration coefficients[J]. IEEE Transactions on Evolutionary Computation, 2004, 8(3): 240-255. 